\title{
Seismic time-frequency spectral decomposition by matching pursuit
}

\author{
Yanghua Wang ${ }^{1}$
}

\begin{abstract}
A seismic trace may be decomposed into a series of wavelets that match their time-frequency signature by using a matching pursuit algorithm, an iterative procedure of wavelet selection among a large and redundant dictionary. For reflection seismic signals, the Morlet wavelet may be employed, because it can represent quantitatively the energy attenuation and velocity dispersion of acoustic waves propagating through porous media. The efficiency of an adaptive wavelet selection is improved by making first a preliminary estimate and then a localized refining search, whereas complex-trace attributes and derived analytical expressions are also used in various stages. For a constituent wavelet, the scale is an important adaptive parameter that controls the width of wavelet in time and the bandwidth of the frequency spectrum. After matching pursuit decomposition, deleting wavelets with either very small or very large scale values can suppress spikes and sinusoid functions effectively from the time-frequency spectrum. This time-frequency spectrum may be used in turn for lithological analysis - for instance, detection of a gas reservoir. Investigation shows that the low-frequency shadow associated with a carbonate gas reservoir still exists, even high-frequency amplitudes are compensated by inverse- $Q$ filtering.
\end{abstract}

\section{INTRODUCTION}

Matching pursuit has been used recently in seismic signal analysis (Wang and Pann, 1996; Castagna et al., 2003; Liu et al., 2004; Liu and Marfurt, 2005). It decomposes a seismic trace into a series of wavelets that belong to a comprehensive dictionary of functions, including Gabor functions. Such wavelets are called time-frequency atoms in the wavelet transform. In this paper, we employ Morlet wavelets as atoms in the matching pursuit decomposition because it is appropriate for seismic waves with energy attenuation and velocity dispersion (Morlet et al., 1982a, b).
The development of matching pursuit aims to overcome the shortcoming of both the window Fourier transform and the wavelet transform (Mallat and Zhang, 1993; Qian and Chen, 1994). A window Fourier transform is not able to describe signal structures with varying size, because all wavelets have a constant scale $(\sigma)$ that is proportional to the window size. On the contrary, a wavelet transform decomposes a signal over time-frequency atoms of varying scales. However, because a wavelet family is built by restricting its frequency parameter to be inversely proportional to the scale $\sigma$, the expansion coefficients in a wavelet frame do not provide precise estimates of the frequency content of waveforms whose Fourier transform is well localized, especially at high frequencies. In matching pursuit, signal structures are represented by wavelets that match their timefrequency signature. This flexible representation is therefore well adapted to signal analysis (Rebollo-Neira and Lowe, 2002; Capobianco, 2003; Andrle et al., 2004; Andrle and Rebollo-Neira, 2006).

In the matching pursuit procedure, in order to properly represent a constituent wavelet, we need to use the following five parameters: the amplitude $(a)$, the time delay $(u)$, the scale $(\sigma)$, the mean frequency $\left(\omega_{m}\right)$, and the phase $(\phi)$. A conventional matching pursuit algorithm is an expensive iteration procedure, selecting these parameters appropriately among a large and redundant dictionary (Mallat and Zhang, 1993). Using complex-trace attributes and derived analytical expressions in various stages improves efficiency. The procedure may be divided into three stages. In stage 1, first exploit the attributes from complex-trace analysis to obtain three parameters $\left(u, \omega_{m}, \phi\right)$ out of five (Barnes, 1993; Liu and Marfurt, 2005), and then search for the fourth parameter $(\sigma)$. Once we obtain preliminary estimates of these four parameters, we refine them in stage 2 by a local optimization within a certain range (Gribonval, 2001). In stage 3, estimate the amplitude of the wavelet $(a)$. Moreover, we derive some analytical expressions that are employed in stage 1 to efficiently search for the fourth parameter, in stage 2 to refine processing and in stage 3 for amplitude estimation.

The scale parameter $\sigma$ is one of the important parameters for constituent wavelets, as it controls frequency bandwidth and also the width of a wavelet in time. However, this free parameter was not considered by spectral decomposition methods based on the win-

Manuscript received by the Editor April 27, 2006; revised manuscript received September 3, 2006; published online November 22 , 2006.

${ }^{1}$ Imperial College London, Centre for Reservoir Geophysics, Department of Earth Science and Engineering, South Kensington, London SW7 2BP, United Kingdom. E-mail: yanghua.wang@imperial.ac.uk.

(C) 2007 Society of Exploration Geophysicists. All rights reserved. 
dowed Fourier transform or the Gabor transform. It was not considered even by some of the matching pursuit implementations-for example, Liu and Marfurt (2005), in which the width of a wavelet was a function of the central frequency, as is the case in a conventional wavelet transform. Taking $\sigma$ as a free parameter not only means a reduced residual (because an additional degree of freedom will reduce the residual), it also means that many more wavelets in the dictionary are considered by matching pursuit. After matching pursuit, by removing wavelets with either very small or very large $\sigma$ values, we can suppress spikes or sinusoid functions effectively from the time-frequency spectrum. Therefore, we refer to it as a $\sigma$ filter, or sigma filter.

The time-frequency spectrum of a seismic trace is a useful attribute in geophysical analysis and lends itself to gas reservoir detection. Castagna et al. (2003) showed compelling examples that gas reservoirs could be identified by low-frequency shadows in a timefrequency spectrum. We conduct an investigation that reveals the existence of the low-frequency shadow associated with a carbonate gas reservoir, even after we have applied an inverse- $Q$ filter to the seismic data to compensate for the high-frequency amplitudes.

\section{MATCHING PURSUIT WITH MORLET WAVELETS}

\section{Three-stage matching pursuit}

Matching pursuit expands a signal over a series of wavelets or atoms, selected from a dictionary composed of Gabor wavelets, supplemented with a canonical basis of discrete Dirac functions and the discrete Fourier basis of complex exponentials (Mallat and Zhang, 1993). A Gabor wavelet is a complex function in the time domain, represented as a product of a complex sinusoid function by a Gaussian envelope (Gabor, 1946). To efficiently evaluate the phase shift $\phi$, we also include it in the following complex-valued Gabor wavelet as

$$
g_{\gamma}(t)=w\left(\frac{t-u}{\sigma}\right) \exp [i(\omega(t-u)+\phi)]
$$

where $w(t)$ is a Gaussian window, $u$ is the time delay (translation), $\sigma$ is the spread in the time axis (scale), $\omega$ is the center (angular) frequency (modulation), and $\phi$ is the phase shift. Thus, we characterize a Gabor wavelet by a set of four parameters, $\gamma=\{u, \sigma, \omega, \phi\}$.

Matching pursuit is implemented iteratively, and each iteration adaptively extracts an optimal form of wavelet $g_{\gamma_{n}}$, where $n$ is the iteration number. After $N$ iterations, a seismic trace $f(t)$ is expanded into the following form:

$$
f(t)=\sum_{n=0}^{N-1} a_{n} g_{\gamma_{n}}(t)+R^{(N)} f
$$

where $a_{n}$ is the amplitude of the $n$th wavelet $g_{\gamma_{n}}, R^{(N)} f$ is the residual, with $R^{(0)} f=f$. In the $n$th iteration of the decomposition, we adopt our three-stage procedure. In stage 1, we estimate the four parameters $\gamma_{n}=\left\{u_{n}, \sigma_{n}, \omega_{n}, \phi_{n}\right\}$ efficiently, but approximately. In stage 2, we update these four parameters for an optimal wavelet $g_{\gamma_{n}}$. Finally, in stage 3 , we estimate the amplitude $a_{n}$. This three-stage procedure is repeated through all iterations.

In stage 1 , estimate three parameters $\left(u_{n}, \omega_{n}, \phi_{n}\right)$ by exploiting complex-trace analysis. For a real seismic trace, perform the Hilbert transform to compose a complex trace, and then derive complextrace attributes (Taner et al., 1979). Inspired by Liu and Marfurt (2005), we set the time of the maximum envelope of the complex trace to be the time delay $u_{n}$, the instantaneous frequency to be the center frequency $\omega_{n}$, and the instantaneous phase to be the phase $\phi_{n}$. Then, we search for the fourth parameter $\sigma_{n}$, the spread in the time axis, using the following equation:

$$
g_{\gamma_{n}}(t)=\arg \max _{g_{\gamma_{n}} \in D} \frac{\left|\left\langle R^{(n)} f, g_{\gamma_{n}}\right\rangle\right|}{\left\|g_{\gamma_{n}}\right\|},
$$

where $D=\left\{g_{\gamma}(t)\right\}_{\gamma \in \Gamma}$ is a comprehensive dictionary of the constituent wavelets, $\langle f, h\rangle$ denotes inner product of functions $f$ and $h$, and $\left\|g_{\gamma_{n}}\right\|=\sqrt{\left\langle g_{\gamma_{n}}, g_{\gamma_{n}}\right\rangle}$ normalizes wavelet $g_{\gamma_{n}}$. We use equation 3 to search for the optimal parameter $\sigma_{n}$ over a group of preselected, uniformly distributed $\sigma$ values with fixed $u_{n}, \omega_{n}$ and $\phi_{n}$ values.

Note that in stage 1 above, we estimate the complex-trace attributes at discrete sampling time and $\sigma$ with discrete value as well. In stage 2, we optimize these four parameters by searching within a subdictionary using equation 3 . The searching range around a parameter $\xi$ is $[\xi-\Delta \xi, \xi+\Delta \xi]$, where $\Delta u$ is the time-sampling interval, $\Delta \sigma$ is the sampling interval used for $\sigma, \Delta \omega$ is the frequency-sampling interval, and $\Delta \phi$ is 5 degrees. This stage of processing is computation-intensive.

Finally, in stage 3, we estimate the amplitude of the optimal wavelet $g_{\gamma_{n}}$ by

$$
a_{n}=\frac{\left|\left\langle R^{(n)} f, g_{\gamma_{n}}\right\rangle\right|}{\left\|g_{\gamma_{n}}\right\|^{2}} .
$$

For the current iteration of decomposition, $R^{(n)} f=a_{n} g_{\gamma_{n}}+R^{(n+1)} f$, where $g_{\gamma_{n}}$ is orthogonal to $R^{(n+1)} f$, we have $\left\|R^{(n)} f\right\|^{2}=\left|\left\langle R^{(n)} f, g_{\gamma_{n}}\right\rangle\right|^{2} /$ $\left\|g_{\gamma_{n}}\right\|^{2}+\left\|R^{(n+1)} f\right\|^{2}$. For the orthogonal projection (equation 3 ), we choose $g_{\gamma_{n}}$ with a maximum $\left|\left\langle R^{(n)} f, g_{\gamma_{n}}\right\rangle\right| /\left\|g_{\gamma_{n}}\right\|$ such that the residual $\left\|R^{(n+1)} f\right\|^{2}$ is minimized. Therefore, equation 4 satisfies the minimum residual condition

$$
\left\{a_{n}, \gamma_{n}\right\}=\arg \min _{a_{n}, \gamma_{n} \in \Gamma}\left\|R^{(n+1)} f(t)\right\|^{2} .
$$

The final minimum residual $R^{(N)} f(t)$, after $N$ iterations, is regarded as the data noise.

\section{Matching pursuit with Morlet wavelets}

For seismic reflection signal analysis, Morlet et al. (1982a, b) used the complex Gabor wavelet (1) as a basic signal model, involving possible time delay because of velocity dispersion and high-frequency attenuation based on a frequency independent- $Q$ model. This Morlet wavelet is suitable for energy and frequency quantification of seismic data and, particularly, is appropriate for attenuation and resolution studies of acoustic waves propagating through porous media.

A Morlet wavelet $m(t)$ centered at the abscissa $u$ can be defined as (Morlet et al., 1982b)

$$
m(t)=\exp \left[-\left(\frac{\ln 2}{\pi^{2}}\right) \frac{\omega_{m}^{2}(t-u)^{2}}{\sigma^{2}}\right] \exp \left[i\left(\omega_{m}(t-u)+\phi\right)\right]
$$

where $\omega_{m}$ is the mean angular frequency, and $\sigma$ is a constant value, which controls the wavelet width. The Morlet wavelet has a constant shape ratio, $($ diameter/mean period $=$ constant $)$, where the diameter or duration is measured at half of the maximum amplitude of the wavelet envelope, or $-6 \mathrm{~dB}$ in logarithmic scale. 
The Fourier transform of $m(t)$ is given as follows:

$$
\begin{aligned}
M(\omega)= & \frac{1}{2 \pi} \int_{-\infty}^{+\infty} \exp \left[-\frac{\ln 2}{\pi^{2}} \frac{\omega_{m}^{2}}{\sigma^{2}}(t-u)^{2}\right. \\
& \left.-i\left(\omega-\omega_{m}\right)(t-u)-i(\omega u-\phi)\right] d t .
\end{aligned}
$$

Using the following definite integral

$$
\int_{-\infty}^{+\infty} \exp \left[-a \tau^{2}-b \tau-c\right] d \tau=\sqrt{\frac{\pi}{a}} \exp \left[\frac{b^{2}}{4 a}-c\right],
$$

we may obtain the frequency spectrum as

$$
\begin{aligned}
M(\omega)= & \frac{1}{2} \sqrt{\frac{\pi}{\ln 2}} \frac{\sigma}{\omega_{m}} \exp \left[-\left(\frac{\pi^{2}}{4 \ln 2}\right) \frac{\sigma^{2}\left(\omega-\omega_{m}\right)^{2}}{\omega_{m}^{2}}\right] \\
& \times \exp [-i(\omega u-\phi)],
\end{aligned}
$$

where $\omega_{m}>0$ is assumed. Note that the Morlet wavelet also has a constant shape ratio (bandwidth/mean frequency $=$ constant $)$ in the frequency domain as well.

From expressions 6 and 9 above, we can estimate the time and frequency extents of a wavelet with mean angular frequency $\omega_{m}$ and width $\sigma$. Half of the time window length and half of the frequency window length are estimated as

$$
\ell_{t}=1.28 \sqrt{|\varepsilon|} \frac{\sigma}{\omega_{m}}, \quad \text { and } \ell_{\omega}=0.18 \sqrt{|\varepsilon|} \frac{\omega_{m}}{\sigma},
$$

where $\varepsilon$ is the minimum amplitude in $\mathrm{dB}$, which in the following examples is set as $\varepsilon=-120 \mathrm{~dB}$. We can see from these expressions that the width in time and in frequency are inversely proportional to each other, and that the widths depend on $\sigma$. The latter property will be utilized in the next section to design a filter.

\section{The time-frequency spectrum}

After decomposing a signal $f(t)$ into a series of wavelets $g_{\gamma_{n}}(t)$, for $n=0,1,2, \ldots, N-1$, we can have a clear picture of the energy distribution in the time-frequency plane. The energy density of signal $f(t)$ in the time-frequency plane is calculated by

$$
E f(t, \omega)=\sum_{n=0}^{N-1} \frac{a_{n}^{2}}{\left\|g_{\gamma_{n}}\right\|^{2}} W g_{\gamma_{n}}(t, \omega),
$$

where $W g_{\gamma_{n}}(t, \omega)$ is the Wigner distribution of a selected wavelet $g_{\gamma_{n}}(t, \omega)$. Similarly, we define here the amplitude spectrum in the time-frequency space as

$$
A f(t, \omega)=\sum_{n=0}^{N-1} \frac{a_{n}}{\left\|g_{\gamma_{n}}\right\|} \sqrt{W g_{\gamma_{n}}(t, \omega)} .
$$

For the Morlet wavelet $m(t)$ given in equation 6, the Wigner distribution in the time-frequency domain is defined by

$$
W g_{\gamma}(t, \omega)=\frac{1}{2 \pi} \int_{-\infty}^{+\infty} m\left(t+\frac{\tau}{2}\right) \bar{m}\left(t-\frac{\tau}{2}\right) \exp [-i \omega \tau] d \tau,
$$

where $\bar{m}(t)$ is the complex conjugate of $m(t)$. Using the definite integral 8 again, we obtain

$$
\begin{aligned}
& W g_{\gamma}(t, \omega)=\sqrt{\frac{\pi}{2 \ln 2}} \frac{\sigma}{\omega_{m}} \exp \left[-\left(\frac{\pi^{2}}{2 \ln 2}\right) \frac{\sigma^{2}\left(\omega-\omega_{m}\right)^{2}}{\omega_{m}^{2}}\right] \\
& \times \exp \left[-\left(\frac{2 \ln 2}{\pi^{2}}\right) \frac{\omega_{m}^{2}(t-u)^{2}}{\sigma^{2}}\right] \text {. }
\end{aligned}
$$

We then have an analytic expression for the time-frequency amplitude spectrum as

$$
\begin{aligned}
A f(t, \omega)= & \sum_{n=0}^{N-1} \frac{a_{n}}{\left\|g_{\gamma_{n}}\right\|}\left(\sqrt{\frac{\pi}{2 \ln 2} \frac{\sigma_{n}}{\omega_{n}}}\right)^{1 / 2} \\
& \times \exp \left[-\left(\frac{\pi^{2}}{4 \ln 2}\right) \frac{\sigma_{n}^{2}\left(\omega-\omega_{n}\right)^{2}}{\omega_{n}^{2}}\right] \\
& \times \exp \left[-\left(\frac{\ln 2}{\pi^{2}}\right) \frac{\omega_{n}^{2}\left(t-u_{n}\right)^{2}}{\sigma_{n}^{2}}\right],
\end{aligned}
$$

where $\omega_{n} \equiv \omega_{m, n}$ is the mean frequency of the $n$th wavelet, and $\left\|g_{\gamma_{n}}\right\|$ is a normalization factor derived in the following subsection.

\section{An efficient implementation}

To speed up the computation, we derive an analytic expression for the module $\left\|g_{\gamma_{n}}\right\|^{2}$.

For the decomposition of a real seismic trace, we use a real-valued wavelet in matching pursuit:

$$
g_{\gamma}(t)=w\left(\frac{t-u}{\sigma}\right) \cos [\omega(t-u)+\phi] .
$$

For such a real wavelet, we formulate the module $\left\|g_{\gamma_{n}}\right\|^{2}$ in terms of the following two integrations,

$$
D_{0}=\int_{-\infty}^{\infty}\left[w\left(\frac{t-u}{\sigma}\right)\right]^{2} d t
$$

$D_{1}(\omega)=\int_{-\infty}^{\infty}\left[w\left(\frac{t-u}{\sigma}\right)\right]^{2} \exp [i 2(\omega(t-u)+\phi)] d t$,

where equation 18 is a discrete Fourier transform plus phase shift, and we calculate it by

$$
\left\|g_{\gamma_{n}}\right\|^{2}=\frac{1}{2}\left(D_{0}+\operatorname{Re}\left[D_{1}(\omega)\right]\right)
$$

Considering a real-valued Morlet wavelet in the form of

$$
m(t)=\exp \left[-\left(\frac{\ln 2}{\pi^{2}}\right) \frac{\omega_{m}^{2}(t-u)^{2}}{\sigma^{2}}\right] \cos \left[\omega_{m}(t-u)+\phi\right]
$$

we may now derive an analytical expression for the module $\left\|g_{\gamma_{n}}\right\|^{2}$. Starting from the two integrations 17 and 18 ,

$$
\begin{gathered}
D_{0}=\frac{\sigma \pi}{\omega_{n}} \sqrt{\frac{\pi}{2 \ln 2}}, \\
D_{1}\left(\omega_{n}\right)=\frac{\sigma \pi}{\omega_{n}} \sqrt{\frac{\pi}{2 \ln 2}} \exp \left[-\frac{\pi^{2} \sigma^{2}}{2 \ln 2}+i 2 \phi\right],
\end{gathered}
$$

we obtain the following analytical expression, 


$$
\left\|g_{\gamma_{n}}\right\|^{2}=\frac{\pi}{2} \sqrt{\frac{\pi}{2 \ln 2}} \frac{\sigma}{\omega_{n}}\left(1+\exp \left[-\frac{\pi^{2} \sigma^{2}}{2 \ln 2}\right] \cos \phi\right) .
$$

This expression may be calculated efficiently and quickly for equations 3 and 4, and for analytical expression 15 as well.

\section{SCALE SIGMA AND SIGMA FILTERING}

Matching pursuits provide extremely flexible signal representations, with the properties given explicitly by the amplitude, time,
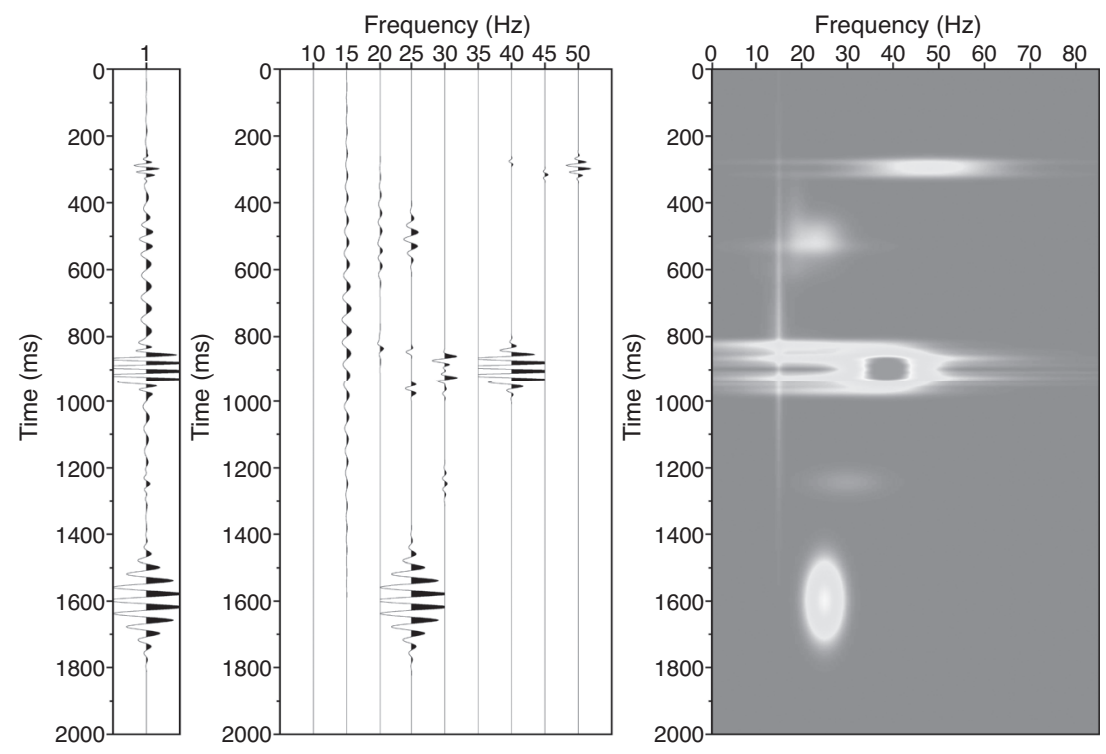

Figure 1. A synthetic seismic trace, decomposed wavelets, and associated amplitude spectrum in the time-frequency domain. In the spectrum, the three wavelets around $300 \mathrm{~ms}$ can be clearly separated in time. Note also a group of wavelets centered at $900 \mathrm{~ms}$ with different mean frequencies. The spectrum suggests at least four wavelets next to each other, which are impossible to distinguish from the seismic trace. However, the spectrum of the wave package centered at $1600 \mathrm{~ms}$ clearly indicates that it is indeed a single wavelet isolated from others.
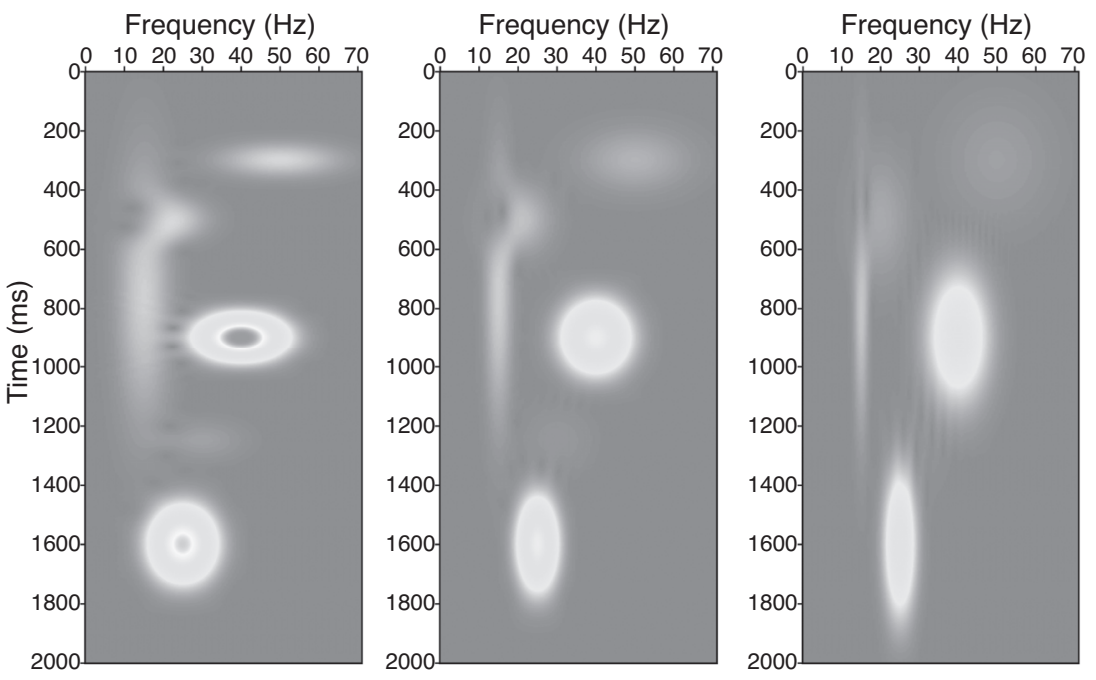

Figure 2. Gabor-transform spectra with different Gaussian window size. The input is the same synthetic trace shown in Figure 1, but the time-frequency spectrum generated by a Gabor transform strongly depends on the given window size. From left to right, the window size used is 100,200 , and $400 \mathrm{~ms}$, respectively. In addition, the resolution in general is lower than the one generated from matching pursuit (shown in Figure 1). scale, frequency, and phase values of the selected wavelets. From a wave package centered at about $900 \mathrm{~ms}$ also is centered at $1600 \mathrm{~ms}$ is indeed a single isolated wavelet.

For a comparison, Figure 2 displays the time-frequency spectra generated by a windowed Fourier transform or Gabor transform presented in Wang (2006), in which a Gaussian window $w(t)$ is defined by

$$
w(t-u)=\frac{2}{T \sqrt{\pi}} \exp \left[-4\left(\frac{t-u}{T}\right)^{2}\right],
$$

where $T$ is the (half) window length. The input is the same synthetic trace of Figure 1. But we can see from Figure 2 that the Gabor-transform spectra strongly depend on the window size given for the transform. In Figure 2, the window size used in each panel from left to right is $(2 T=) 100$, 200, and $400 \mathrm{~ms}$, respectively. In addition, the resolution is lower than the one in Figure 1, generated from matching pursuit. For example, the same wave package centered at $1600 \mathrm{~ms}$ appears to be different in the time-frequency spectra with different window size. Between 400 and $600 \mathrm{~ms}$, a wavelet at $500 \mathrm{~ms}$ and a $15-\mathrm{Hz}$ sinusoid cannot be separated properly by the Gabor transform using either short or long window size.

In our matching pursuit scheme, the scale parameter $\sigma$ that controls the width of a wavelet (and the frequency band) is selected adaptively. Therefore, it can even extract spikes and sinusoids. For example, in Figure 1, the time-frequency spectrum displays a sinusoid of $15 \mathrm{~Hz}$ within the 100-1500 ms time window. The time-frequency spectrum can separate neighboring wavelets that are impossible to distinguish from the signal trace. The three wavelets around $300 \mathrm{~ms}$ can be clearly identified from the spectrum. One is an ellipse and two are band-limited spikes.

The amplitude distribution of wavelets is visible as vertical lines for cosine functions, horizontal lines for spike (Dirac $\delta$ ), or ellipses with axes proportional to time and frequency spread for $\mathrm{Ga}$ bor functions. Therefore, based on the time-frequency spectrum, we may design a filter to reject certain wavelets, such as the cosine functions, and spikes. We propose to filter decomposed wavelets or the time-frequency spectrum based on the $\sigma$ value, the so-called sigma filter.

Figure 3 displays a real seismic trace that might be a good representative for land seismic data, decomposed wavelets, and the time-frequency domain amplitude spectrum, in which we see spikes and sinusoid functions. In the central panel above, the frequency value on the top axis is the central frequency of a frequency range. For example, the $50-\mathrm{Hz}$ trace consists of wavelets with mean frequencies between 47.5 and $52.5 \mathrm{~Hz}$. 

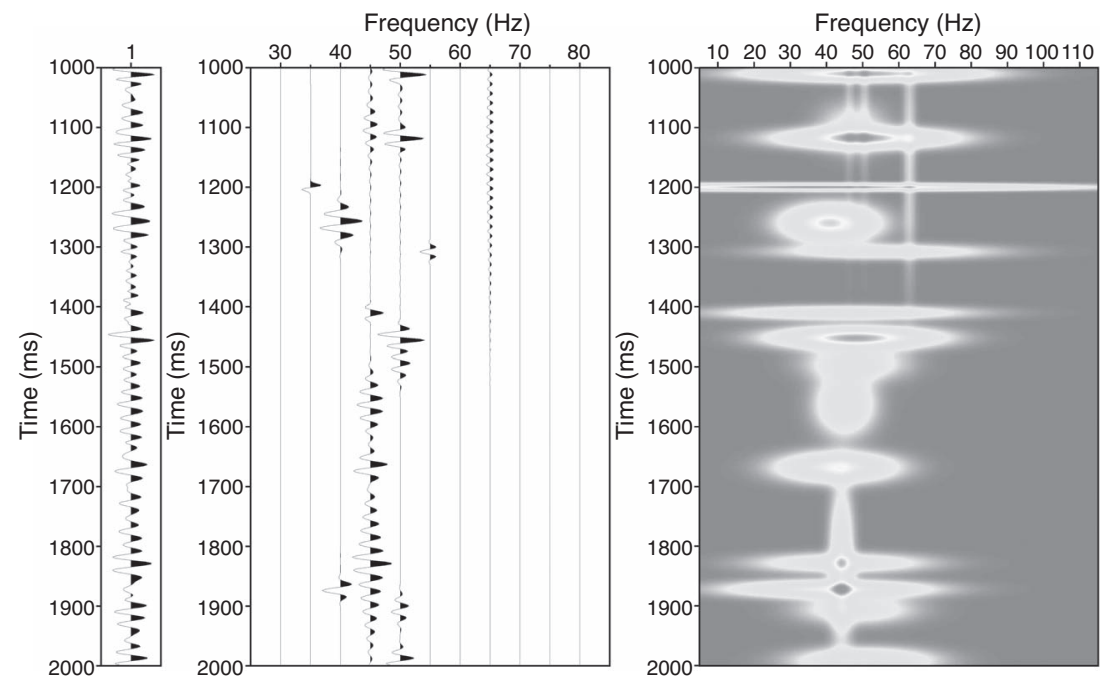

Figure 3. A real seismic trace, decomposed wavelets with mean frequency at different ranges, and the corresponding amplitude spectrum in the time-frequency space. The frequency value on the top axis is the center of a mean-frequency range. For example, the $50-\mathrm{Hz}$ trace consists of wavelets with mean frequencies between 47.5 and $52.5 \mathrm{~Hz}$.
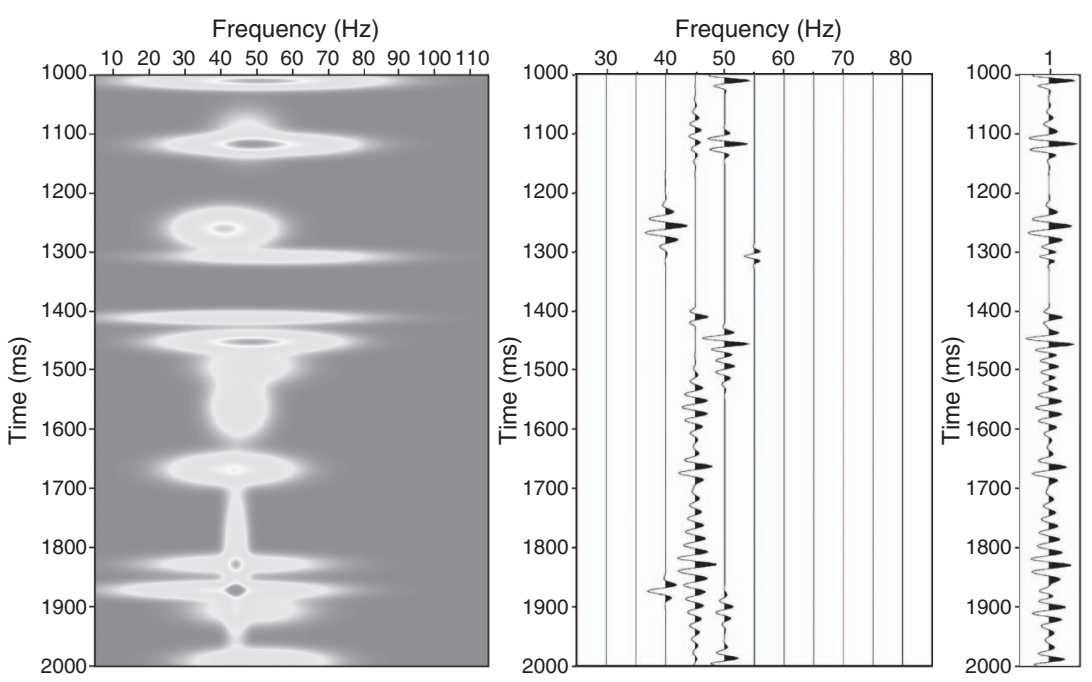

Figure 4. The result of sigma filtering. The time-frequency amplitude spectrum, corresponding wavelets with mean frequency at different ranges, and reconstructed seismic trace after the so-called sigma filter. In this example, the low cutoff is $\sigma=0.4$, and the high cutoff is $\sigma=10$.

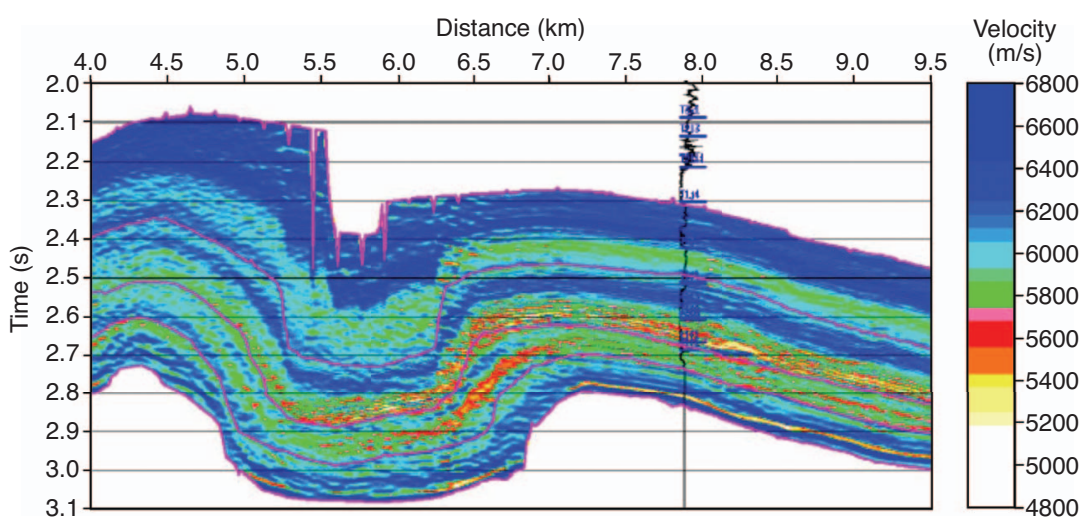

Figure 5. A velocity inversion profile shows the target carbonate gas reservoir with strong heterogeneity at 2.55-2.90 s with a spatial coverage between 6.5 and $9.5 \mathrm{~km}$.
The two expressions in equation 10 suggest that for a small $\sigma$ value, the wavelet represents a spike that has a broad frequency band and short duration in time; and for a large $\sigma$ value, it represents a sinusoid function that has a narrow frequency band and long duration in time. Therefore, in this example, we reject wavelets with $\sigma$ $<0.4$ and wavelets $\sigma>10$ and produce the result shown in Figure 4: the time-frequency amplitude spectrum, corresponding wavelets with different mean frequencies, and reconstructed seismic trace after sigma filtering. Using sigma filtering, we may clean up the time-frequency spectrum for the geophysical analysis, such as gas reservoir detection.

\section{APPLICATION TO A GAS RESERVOIR}

Now we apply the adaptive time-frequency decomposition method to a real seismic data example with a target carbonate gas reservoir. Figure 5 shows a velocity inversion profile of the target reservoir between 2.55 and $2.90 \mathrm{~s}$ in time. It has a strong heterogeneity with a spatial coverage between 6.5 and $9.5 \mathrm{~km}$. Because we know where the gas reservoir is, we do expect to see a socalled low-frequency shadow underneath the reservoir. What we try to investigate here is whether the low-frequency shadow would still exist if we apply an inverse- $Q$ filter to the seismic data before performing time-frequency spectral analysis.

Figure 6a displays the seismic section without inverse- $Q$ filtering. We can see that reflection wavelets are lengthened gradually along with increased traveltime or depth in this original seismic section because of the attenuation of highfrequency plane waves. Inverse- $Q$ filtering techniques may compensate for the amplitude dissipation of high-frequency plane waves and correct the phase distortion resulting from frequency dispersion, by which high-frequency plane waves travel faster than low-frequency waves (Wang, 2002, 2006). In Figure 6b, the seismic section after inverse- $Q$ filtering, we do see an improvement on the seismic resolution. After inverse- $Q$ filtering, the width of reflected wavelets becomes consistent from shallow to deep. The resolution improvement also leads to the clear appearance of many subtle faults in the profile. The subtle faults appear above and beneath the target reflection, but the latter is clearly a continuous reflection, and a perfect seal for a gas reservoir. The velocity inversion profile shown in Figure 5 was inverted from the inverse- $Q$ filtered seismic section.

The Morlet wavelet properly represents the energy absorption and phase distortion of wave propagation in porous media (Morlet et al., $1982 \mathrm{a}, \mathrm{b})$ and thus is appropriate for acoustic property analysis of gas reservoirs. In Figure 7 , 

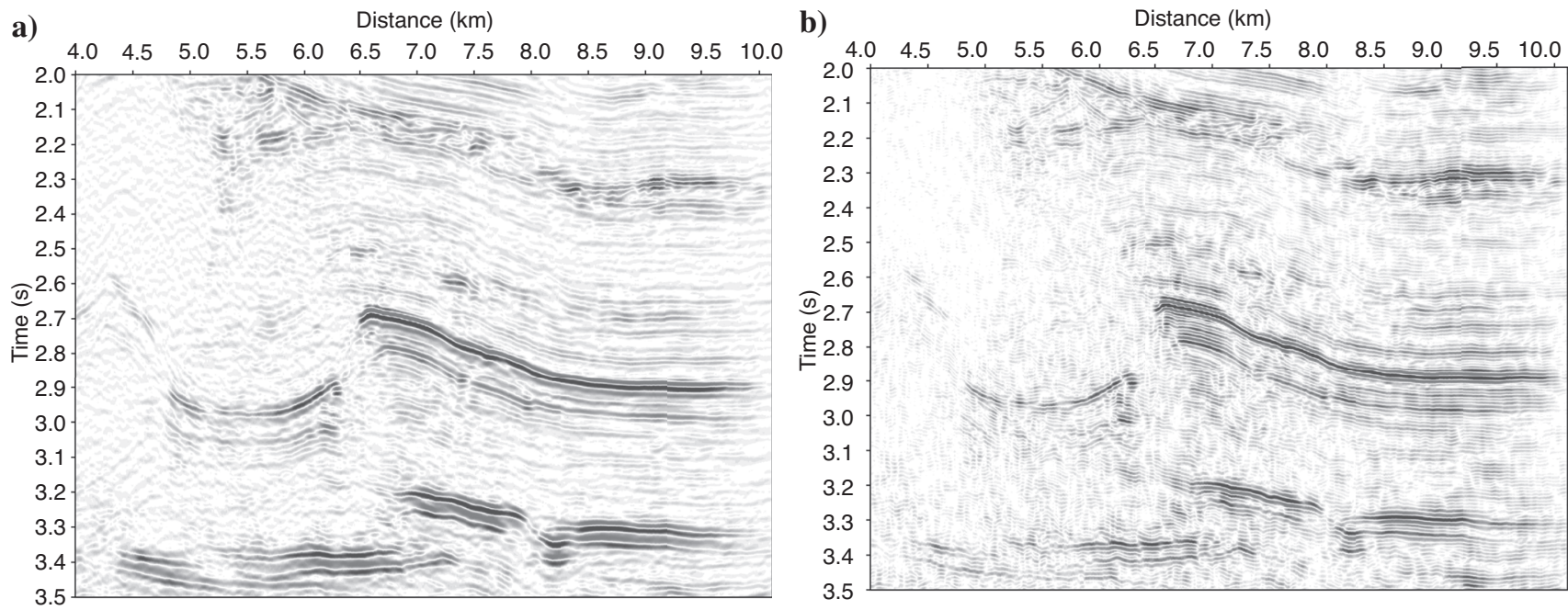

Figure 6. (a) The example seismic profile without inverse- $Q$ filtering, and (b) the section after inverse- $Q$ filtering. After inverse- $Q$ filtering, the width of reflected wavelets becomes consistently narrow from shallow to deep. Many subtle faults appear above and beneath the target reflection. But the latter clearly is a continuous reflection, a perfect seal for a gas reservoir.

Distance $(\mathrm{km})$

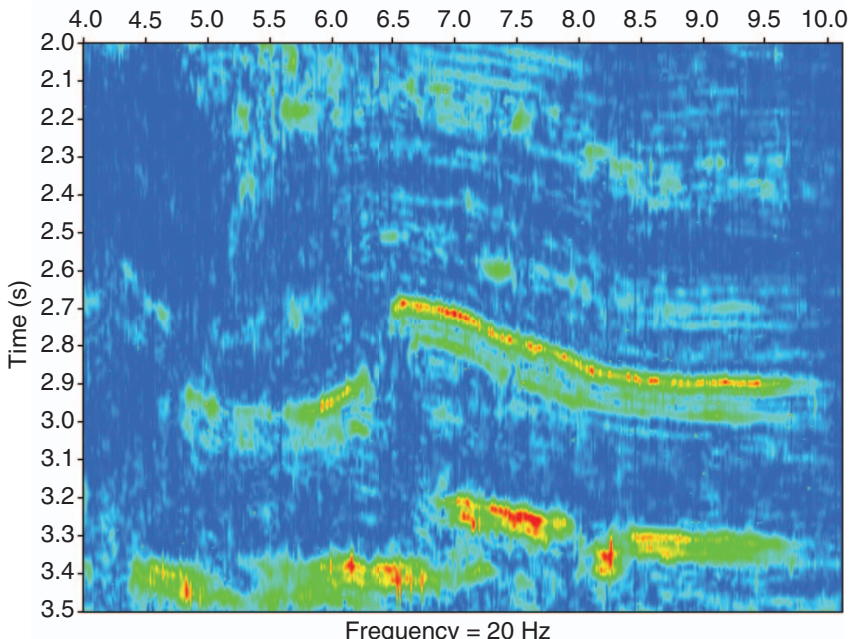

Distance $(\mathrm{km})$

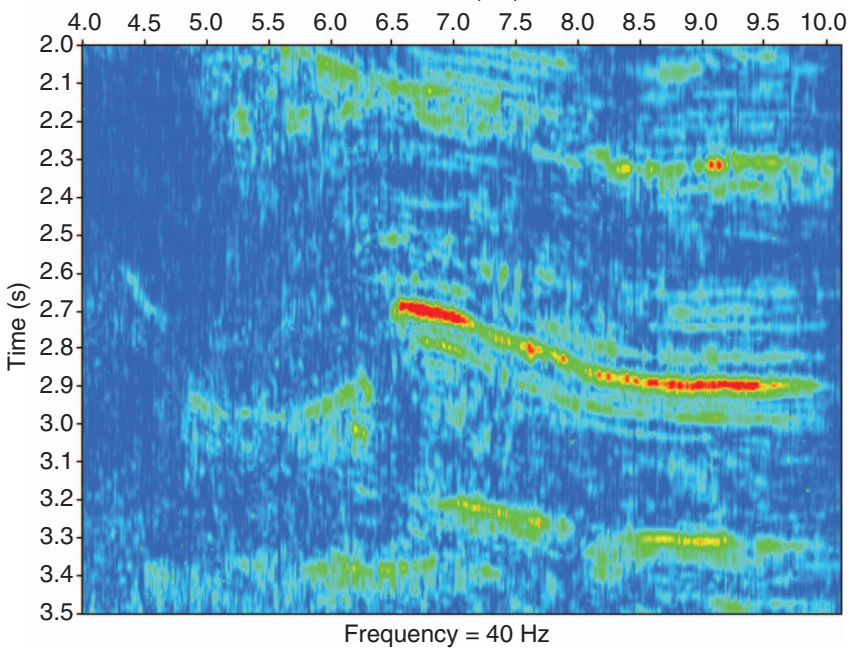

Distance $(\mathrm{km})$

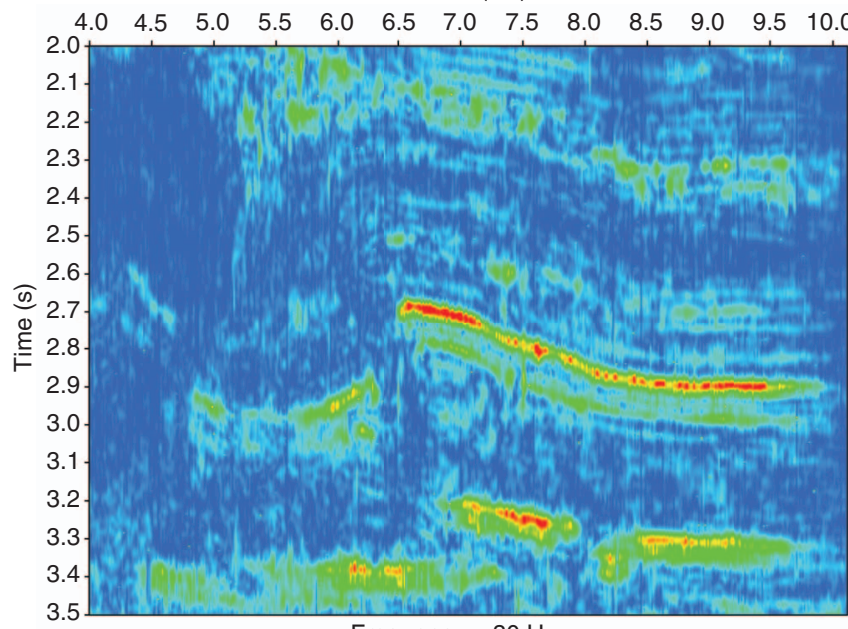

Frequency $=30 \mathrm{~Hz}$

Distance $(\mathrm{km})$

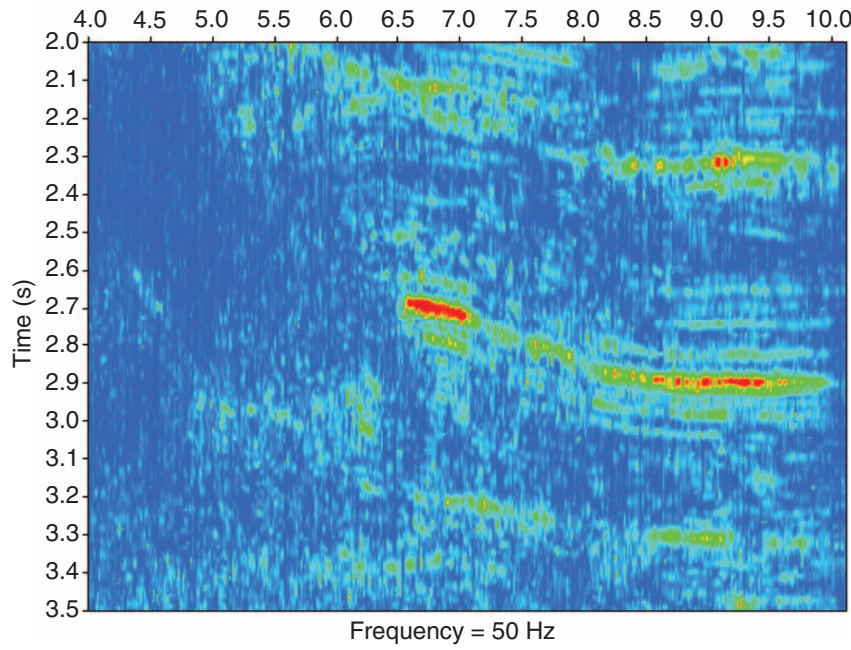

Figure 7. Constant-frequency profiles without inverse- $Q$ filtering. The low-frequency shadow (at 3.0-3.4 s) underneath the carbonate gas reservoir appears in low frequency profiles $(20$ and $30 \mathrm{~Hz})$ but gradually disappears from the high frequency profiles $(40 \mathrm{and} 50 \mathrm{~Hz})$. 

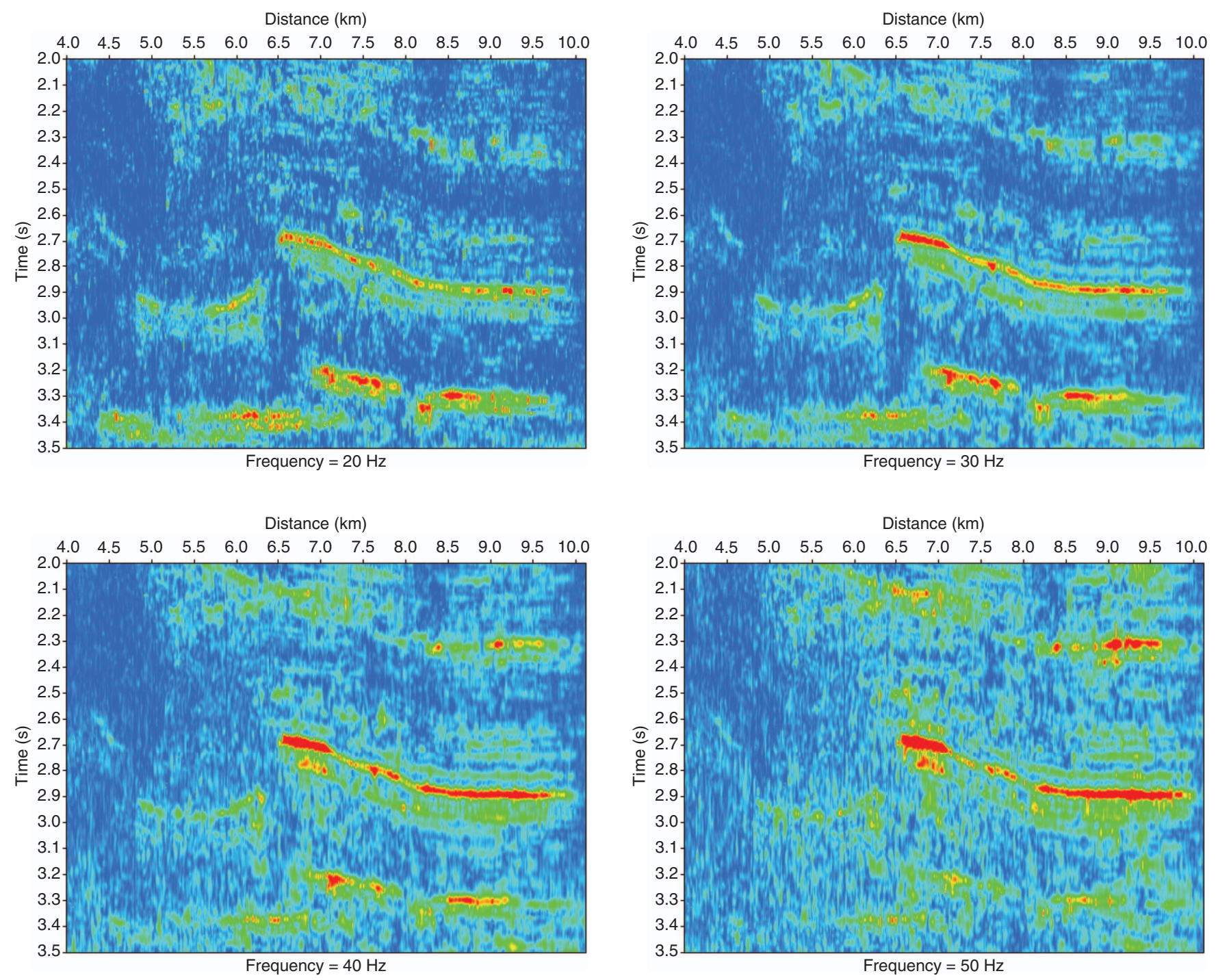

Figure 8 . The constant frequency profiles of the inverse- $Q$ filtered seismic section. The low-frequency shadow zone still exists, and the high-frequency components within that zone were not boosted by amplitude compensation.

we display a group of constant-frequency sections for different frequencies, obtained from the seismic data without inverse- $Q$ filtering (Figure 6a). The gas shadow between 3.0 and $3.4 \mathrm{~s}$, underneath the carbonate gas reservoir, does appear in low-frequency profiles (20 and $30 \mathrm{~Hz}$ ), but gradually disappears in high-frequency profiles (40 and $50 \mathrm{~Hz}$ ). At $20 \mathrm{~Hz}$, the amplitudes in the shadow are even stronger than the amplitudes of the reservoir reflections on the top. As pointed out by Castagna et al. (2003), the shadow is not necessarily a simple attenuation phenomenon because low-frequency energy must have been added or amplified by some physical or numerical process. Attenuation alone should simply decrease higher frequencies, not boost lower frequencies. More examples for time-frequency decomposition can be found in Chakraborty and Okaya (1995), Castagna et al. (2003), and Sinha et al. (2005).

Figure 8 displays the constant-frequency profiles obtained from the inverse- $Q$ filtered seismic section (Figure 6b). Inverse- $Q$ filtering will, in general, boost the amplitudes of high frequencies (Wang, 2002, 2006). But even so, the so-called low-frequency shadow zone still appears in the time-frequency spectrum. It suggests that the dis- appearance of high frequencies around the shadow zone is not caused by earth attenuation, whereas the strong low-frequency amplitudes could be caused by wave interference in a reservoir. Physical explanation of such a shadow zone is an unsolved research topic. Recently, Korneev et al. (2004) tried to explain this low-frequency shadow phenomena using a "frictional-viscous" model (Goloshubin and Bakulin, 1998; Goloshubin and Korneev, 2000; Goloshubin et al., 2001, Goloshubin et al., 2002).

Note that it is possible that the disappearance of higher frequency components for the event below the carbonate reservoir is not a shadow effect, but simply results from different lithologies above and below the reservoir. In the example shown here, we know of the existence of a carbonate gas reservoir and then see the "shadow zone" in the spectrum, but the investigation is not conclusive.

\section{CONCLUSIONS}

Matching pursuit for time-frequency decomposition is an adaptive but expensive iterative approach. We have improved its efficien- 
cy by using (1) complex-trace attributes for a preliminary estimation of parameters for constituent wavelets and (2) derived analytical expressions for efficient computations on the fly. The scale $\sigma$ of a wavelet is an important parameter that controls the width of a wavelet and its frequency bandwidth. By selecting wavelets with different $\sigma$ values, we can effectively filter out the unwanted spikes and sinusoid functions from the time-frequency spectrum. After the so-called sigma filtering, the spectrum can be used in signal analysis. As an example, we have applied it to a real seismic profile that shows the existence of a low-frequency shadow related to a carbonate gas reservoir, even after high-frequency amplitude compensation with inverse- $Q$ filtering.

\section{ACKNOWLEDGMENTS}

I am grateful to the sponsors of the Centre for Reservoir Geophysics, Imperial College London. I also acknowledge Y. Luo and two anonymous reviewers for their constructive comments and suggestions.

\section{REFERENCES}

Andrle, M., and L. Rebollo-Neira, 2006, A swapping-based refinement of orthogonal matching pursuit strategies: Signal Processing, 86, 480-495.

Andrle, M., L. Rebollo-Neira, and E. Sagianos, 2004, Backward-optimized orthogonal matching pursuit approach: IEEE Signal Process Letters, 11, 705-708.

Barnes, A. E., 1993, Instantaneous spectral bandwidth and dominant frequency with applications to seismic reflection data: Geophysics, 58, 419428.

Capobianco, E., 2003, Independent multiresolution component analysis and matching pursuit: Computational Statistics \& Data Analysis, 42, 385-402.

Castagna, J. P., S. J. Sun, and R. W. Siegfried, 2003, Instantaneous spectral analysis: Detection of low-frequency shadows associated with hydrocarbons: The Leading Edge, 22, 120, 122, 124-127.

Chakraborty, A., and D. Okaya, 1995, Frequency-time decomposition of seismic data using wavelet based methods: Geophysics, 60, 1906-1916.

Gabor, D., 1946, Theory of communication: Journal of the Institute of Elec- trical Engineers, 93, 429-457.

Goloshubin, G. M., and A. V. Bakulin, 1998, Seismic reflectivity of a thin porous fluid saturated layer versus frequency: 68th Annual International Meeting, SEG, Expanded Abstracts 976-979.

Goloshubin, G. M., T. M. Daley, and V. A. Korneev, 2001, Seismic low-frequency effects in gas reservoir monitoring VSP data: Presented at the 71st Annual International Meeting, SEG.

Goloshubin, G. M., and V. A. Korneev, 2000, Seismic low-frequency effects from fluid saturated reservoir: 70th Annual International Meeting, SEG, Expanded Abstracts, 1671-1674.

Goloshubin, G. M., V. A. Korneev, and V. M. Vingalov, 2002, Seismic lowfrequency effects from oil-saturated reservoir zones: Presented at the 72nd Annual International Meeting, SEG.

Gribonval, R., 2001, Fast matching pursuit with a multiscale dictionary of Gaussian chirps: IEEE Transactions on Signal Processing, 49, 994-1001.

Korneev, V. A., G. M. Goloshubin, T. M. Daley, and D. B. Silin, 2004, Seismic low frequency effects in monitoring fluid-saturated reservoirs: Geophysics, 69, 522-532.

Liu, J., and K. J. Marfurt, 2005, Matching pursuit decomposition using Morlet wavelet: 75th Annual International Meeting, SEG, Expanded Abstracts, 786-789.

Liu, J., Y. Wu, D. Han, and X. Li, 2004, Time-frequency decomposition based on Ricker wavelet: 74th Annual International Meeting, SEG, Expanded Abstracts, 1937-1940.

Mallat, S., and Z. Zhang, 1993, Matching pursuit with time-frequency dictionaries: IEEE Transactions on Signal Processing, 41, 3397-3415.

Morlet, J., G. Arens, E. Fourgeau, and D. Giard, 1982a, Wave propagation and sampling theory: Part I, Complex signal and scattering in multilayered media: Geophysics, 47, 203-221.

- 1982b, Wave propagation and sampling theory: Part II, Sampling theory and complex waves: Geophysics, 47, 222-236.

Qian, S., and D. Chen, 1994, Signal representation via adaptive normalized Gaussian functions: Signal Processing, 36, 1-11.

Rebollo-Neira, L., and D. Lowe, 2002, Optimized orthogonal matching pursuit approach: IEEE Signal Process Letters, 9, 137-140.

Sinha, S., P. S. Routh, P. D. Anno, and J. P. Castagna, 2005, Spectral decomposition of seismic data with continuous-wavelet transform: Geophysics, 70, P19-P25.

Taner, M. T., F. Koehler, and R. E. Sherrif, 1979, Complex seismic trace analysis: Geophysics, 44, 1041-1063.

Wang, B., and K. Pann, 1996, Kirchhoff migration of seismic data compressed by matching pursuit decomposition: 66th Annual International Meeting, SEG, Expanded Abstracts, 1642-1645.

Wang, Y., 2002, A stable and efficient approach of inverse $Q$ filtering: Geophysics, 67, 657-663

, 2006, Inverse $Q$ filtering for seismic resolution enhancement: Geophysics, 71, no. 3, V51-V60. 